\title{
Der Sanitätsdienst der Patrouille des Glaciers 2022
}

\section{Raphaël Kohlprath}

Major, Chefarzt, Stab Kommando Patrouille des Glaciers, Facharzt für Orthopädische Chirurgie und Traumatologie des Bewegungsapparates

Seit über einem halben Jahrhundert organisiert die Armee die Patrouille des Glaciers, um ihre Truppen im Hochgebirgseinsatz auszubilden. Im Laufe der Jahre wurde dieses Rennen für internationale militärische und zivile Patrouillen geöffnet und zu einer Veranstaltung von internationaler Bedeutung. Der Sanitätsdienst vor Ort ist beträchtlich: 2018 waren 54 medizinische und 30 paramedizinische Fachkräfte im Einsatz. Ein Einblick in diesen medizinischen Dienst.

Nach vielen strukturellen Reorganisationsphasen und der Gründung eines Vereins wird die Patrouille des Glaciers (PdG) weiterhin durch das VBS (Eidgenössisches Departement für Verteidigung, Schutz und Sport) befohlen und durch das Kommando Operationen geleitet. Die PdG ist inzwischen Teil des Weltcupzirkus des Ski-Alpinismus mit dem Hauptmerkmal, dass sie hauptsächlich die längste zu absolvierende Route im ganzen Weltcup hat und hauptsächlich in der Nacht stattfindet (Abb. 1).

Die letzte Ausgabe der PdG im Jahr 2018 hiess mehr als 5700 Patrouillen oder 1900 Seilschaften aus 30 verschiedenen Ländern auf über 3000 Höhenmetern willkommen. Aufgrund dieses Umfangs und der eingesetzten Mittel ist der Sanitätsdienst heute komplexer denn je. Die Topographie und die Streckenlänge führen dazu, dass der Sanitätsdienst auf mehrere Stand-

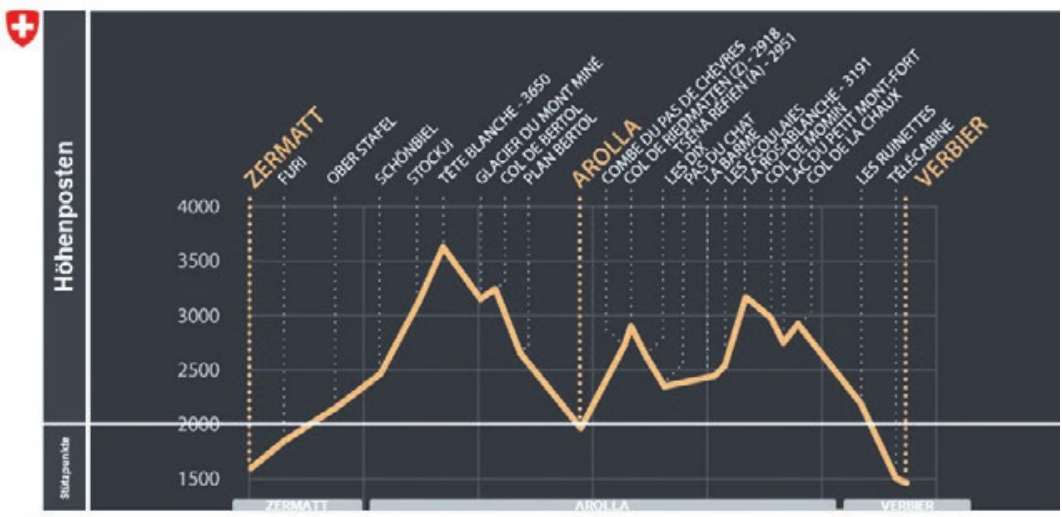

Abbildung 1: Streckenprofil der PdG 2022. Das Rennen hat eine Länge von 57,5 km mit 4386 Höhenmetern positiver Steigung und 4520 Höhenmetern negativer Steigung, das entspricht einer effektiven Leistung von $100 \mathrm{~km}$. Der grösste Teil wird nachts gelaufen. orte verteilt werden muss. Die eingesetzten Truppen müssen autark arbeiten können, da sie unter Umständen aufgrund von schlechtem Wetter im Hochgebirge über mehrere Tage ihre Posten nicht verlassen kön-

\section{Erste Ausgabe der Patrouille}

\section{des Glaciers 1943}

Die Patrouille des Glaciers hat ihren Ursprung in den unruhigen Jahren des Zweiten Weltkrieges. Es ging darum, die Gebirgsbrigade 10 auf die Verteidigung des südwestlichen Teils der Schweizer Alpen vorzubereiten. Bei der ersten Ausgabe kamen wegen katastrophaler Wetterbedingungen nur sechs von zwölf Patrouillen pünktlich in Verbier an.

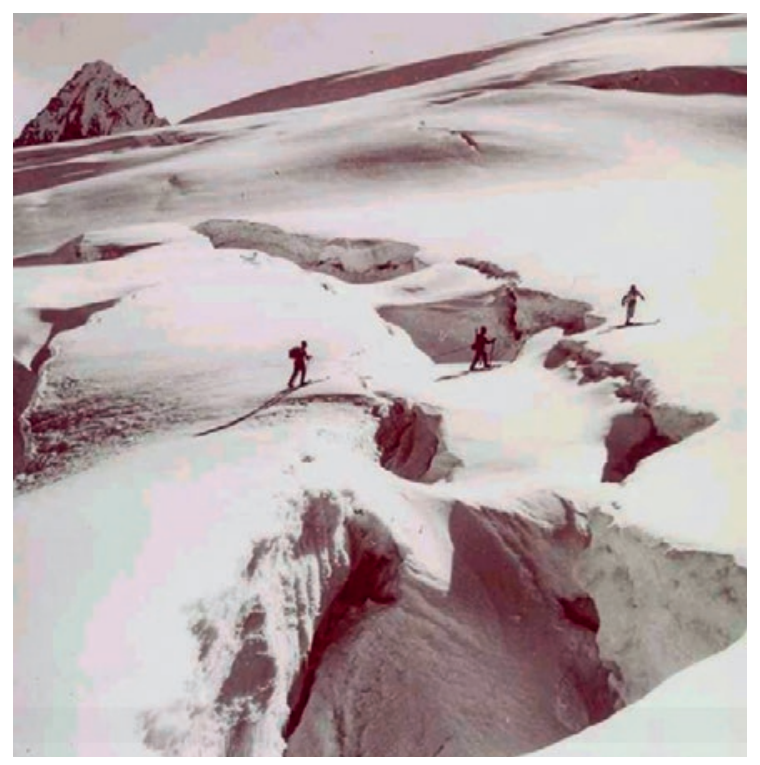

Gletscherspalte von Valpelline im Frühling 1943. 


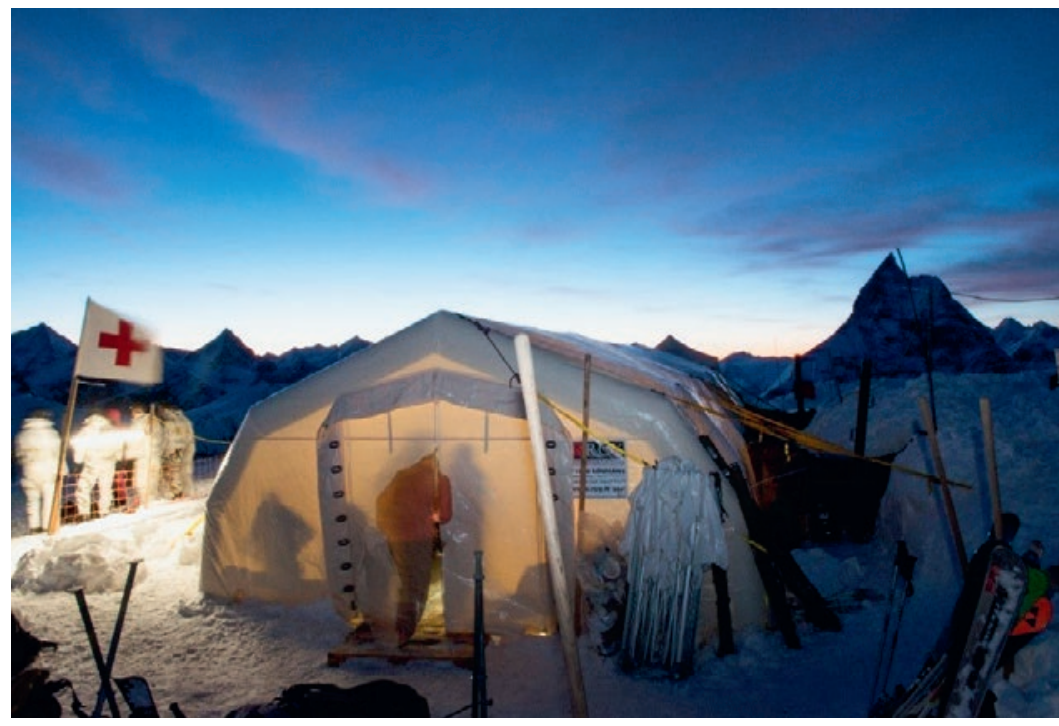

Abbildung 2: Patientensammelpunkt von Tête Blanche, 3650 m ü.M. Diese mythische Passage bleibt vielen Teilnehmenden als kältester Ort in Erinnerung.

nen. Ausserdem muss die Evakuation mittels Helikopter an schwer zugänglichen Stellen gewährleistet werden (Abb. 2).

Während der PdG 2018 waren 54 Ärzte und 30 Sanitäter für acht Tage auf den Höhenposten im Einsatz (Abb. 3). Typischerweise werden pro Ausgabe der PdG 140 verletzte Teilnehmende und etwa sechs Tonnen medizinisches Material und Gerätschaften mit den Helikoptern transportiert.

Da die statistischen Daten zu heterogen sind, ist ein epidemiologischer Vergleich zwischen den Ausgaben derzeit nicht möglich. Trotz allem gibt die PdG 2014 ein gutes Beispiel des Profils der üblicherweise anzutreffenden Pathologien. Einhundertzweiundsiebzig Fälle wurden während der beiden Rennen behandelt, die sich wie folgt gemäss dem National Advisory Committee of Aeronautics Score (NACA) [1] verteilen: 22 NACA-0, 143 NACA-I, 2 NACA-II, 3 NACA-III, 1 NACA-IV, 1 NACA-V. Die häufigsten Fälle (NACA-I) sind in Tabelle 1

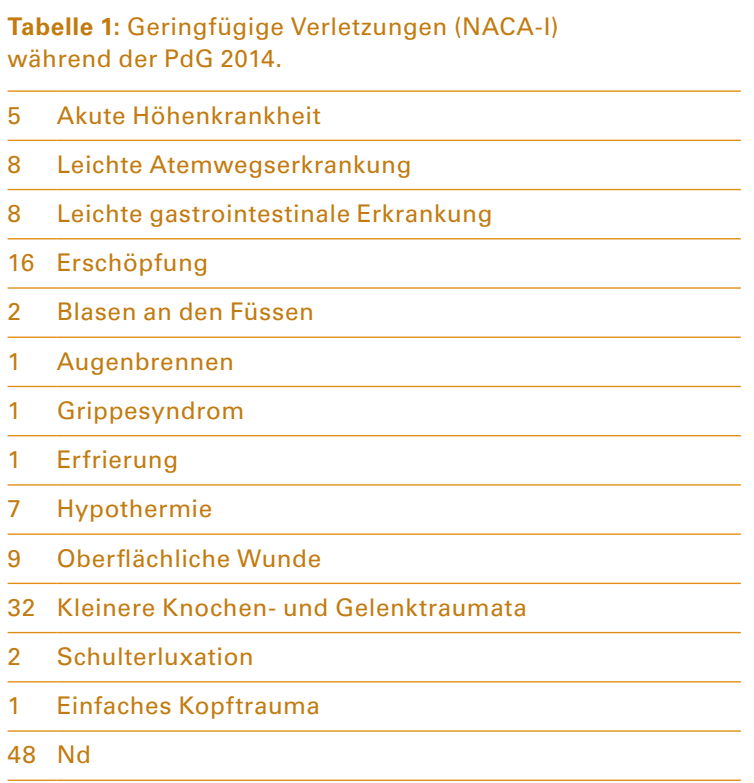

aufgeführt. Schwerwiegendere Fälle (NACA-II bis -V) umfassen Frakturen, akutes Abdomen, Lungenembolie und akutes Lungenödem.

Das Auswahlverfahren für die PdG 2022 wird durch die hohen Anforderungen sehr anspruchsvoll. Die eingesetzten Ärztinnen und Ärzte und das Pflegepersonal profitieren drei Monate vor Start der PdG von einer einsatzbezogenen Ausbildung (EBA), um die für das Hochgebirge benötigten medizinischen und technischen Fähigkeiten zu vertiefen. An dieser Schulung erhält das eingesetzte Sanitätspersonal eine Ausbildung in Gebirgsmedizin, Gebirgstrauma, dem Verhalten bei Helikopter-Evakuierungen (EC-635) und Bergrettungstechniken. Diese Ausbildungen werden von den wichtigsten Partnern der PdG durchgeführt, u.a. von der Luftwaffe und von den Bergführern des Kompetenzzentrums Andermatt.

Um eine hohe Kompetenz im Sanitätsdienst zu erhalten, suchen wir sowohl ärztliche als auch paramedizi-

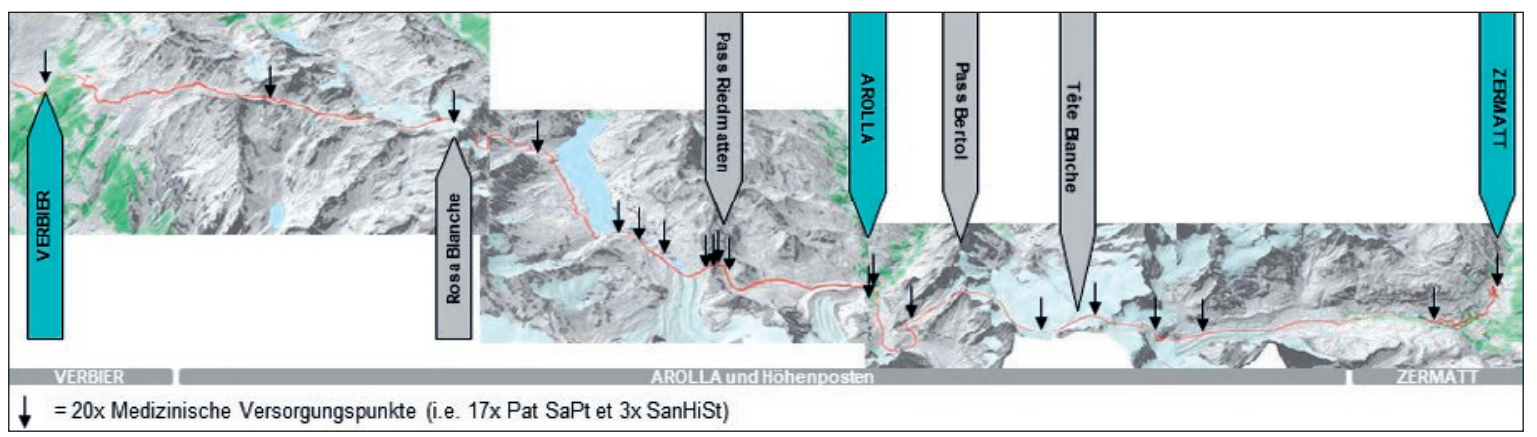

Abbildung 3: Vereinfachtes Schema des Sanitätsdispositivs der PdG 2022. Die Höhenposten befinden sich im Hochgebirge, das entlang der Alpenhöhenroute (im Durchschnitt 3000 Höhenmeter) verläuft. Schwarze Pfeile stellen medizinische Versorgungspunkte dar. 


\section{Erfahrungsbericht von Christophe Chablais, 15 Jahre Erfahrung in der PdG}

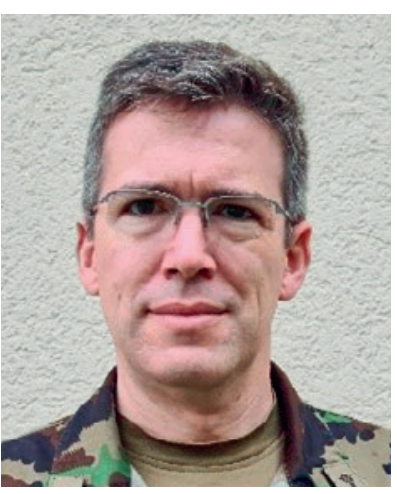

Oberleutnant Christophe Chablais

S4 Sanität, Stab Kommando Patrouille des Glaciers

Rettungssanitäter im CSU von Waadt Nord und la Broye.

Nach meiner Ausbildung zum Kompanieführer werde ich im Jahr 2005 in der Notrufzentrale der Sanität eingeteilt. Meine Aufgabe ist es, Hilferufe der Patrouillen entgegenzunehmen und die Kommunikation zwischen den sanitätsdienstlichen Elementen sicherzustellen. Als Rettungssanitäter im zivilen Leben konnte ich durch diese erste Erfahrung die Herausforderungen, die die Kollegen in der 144-Zentrale im Alltag erleben, besser verstehen.
Anschliessend hatte ich die Gelegenheit, die Verwaltung des gesamten Sanitätsmaterials zu übernehmen, d.h. etwa sechs Tonnen Material, das durch die Schweiz geschickt und anschliessend auf den Höhenposten verteilt wird. In diesen fünfzehn Jahren hat meine Rolle an Bedeutung gewonnen, und viele Herausforderungen mussten gemeistert werden. Meine Ratschläge wurden oft berücksichtigt und meine zivilen Fähigkeiten miteinbezogen. Zum Beispiel wurden standardisierte Prozesse entwickelt, welche auf den Sanitätsposten individuell eingesetzt und angepasst wurden. Dadurch konnten der Materialbeschaffungsprozess optimiert und der Materialverlust reduziert werden. Die oft extremen Temperaturbedingungen schränkten die Lagerbedingungen für Schlüsselgüter wie Medikamente ein, die bei Ende der PdG oft vernichtet werden müssen. Dank dieser Massnahmen konnte in der Ausgabe 2018 der Verbrauch von Sanitätsmaterial um $25 \%$ gesenkt werden.

Die PdG ermöglichte es mir auch, meine Fähigkeiten zu vertiefen, und beeinflusste mein ziviles Berufsleben, sowohl als Logistikoffizier als auch als Einsatzleiter im Advanced Medical Post Detachment in Lausanne. Besonders im Einsatz bei Air14 und beim Schwingfest in Estavayer-le-Lac konnte ich meine Erfahrungen aus der PdG anwenden. Ich freue mich, Teil dieses grossen Abenteuers zu sein, und hoffe, bald die richtige Person zu finden, an die ich die Fackel weitergeben kann und die bereit ist, für mehrere Ausgaben der PdG diese wichtige Aufgabe zu übernehmen.

\section{Erfahrungsbericht von Christophe Bianchi, Arzt PdG 2016}

Die PdG medizinisch zu unterstützen bedeutet, an einem legendären Rennen in einer einzigartigen Umgebung teilzunehmen und Emotionen von seltener Intensität zu erleben. Meine Verbindung zur PdG begann 2014 als Teilnehmer, nachdem mich ein Freund dazu motiviert hat, am grossen Rennen teilzunehmen. Ein Jahr später, nach über zehn Rennstunden, fällt mir nur ein Gedanke ein, als ich den Aufstieg der Rosablanche sah: «Warum machst du das nur?» Trotz der Anstrengung und der Zweifel, glücklich und müde, aber vor allem pünktlich, überquerten wir die Ziellinie. Diese Erfahrung hat mein Leben geprägt: Ich habe zwar viel über die Berge, aber vor allem viel über mich selbst gelernt.

Zwei Jahre später sind wir wieder am Berg Rosablanche, diesmal aber als Ärzte. Nach dem Aufstieg von Verbier mit den Tourenski, beladen mit unserer Militärausrüstung, entdecken wir das Zelt, in dem wir eine Woche voller Überraschungen verbringen werden. Von den Soldaten des Gebirgsinfanteriebataillons mit einem Glas Weisswein begrüsst, bleibt uns gerade noch Zeit, uns hinzusetzen und etwas zu essen, bevor wir uns auf den Weg machen, die Stufen des mythischen Anstiegs der Rosablanche aus dem Eis zu schlagen. Nachdem ich den Berg vor zwei Jahren bestiegen habe, weiss ich, wie wichtig diese Stufen sind. Entsprechend sogfältig bin ich dabei und denke mir, dass ich dadurch den Teilnehmern beim Rennen ein wenig helfen kann.

Nach einem majestätischen Sonnenuntergang verbringen wir die erste Nacht in unserem Zelt. Man schläft fantastisch auf über 3200 Metern! Nach einem Tag der Vorbereitungen und einer Skitour gehen wir früh ins Bett. Tatsächlich werden die ersten Patrouillen um 21.45 Uhr Zermatt verlassen. Wir müssen also bereit sein, sie am frühen Morgen noch vor Sonnenaufgang willkommen zu heissen.

Am Fusse des Rosablanche, um 4.30 Uhr morgens, sah ich in der Ferne etwas, was ich nie vergessen werde. Mitten in den Bergen in einer sternenklaren Nacht kam uns eine lange Lichterschlange von Stirnlampen entgegen. Es waren die ersten Patrouillen, welche dieses unglaublich schöne Bild abgaben. Was für eine unbeschreibliche Magie. Dann nimmt alles Fahrt auf: Die Patrouillen ziehen vorbei, und wir treffen uns am frühen Nachmittag, als das Ende des Rennens bekanntgegeben wurde. Was für ein Abenteuer!

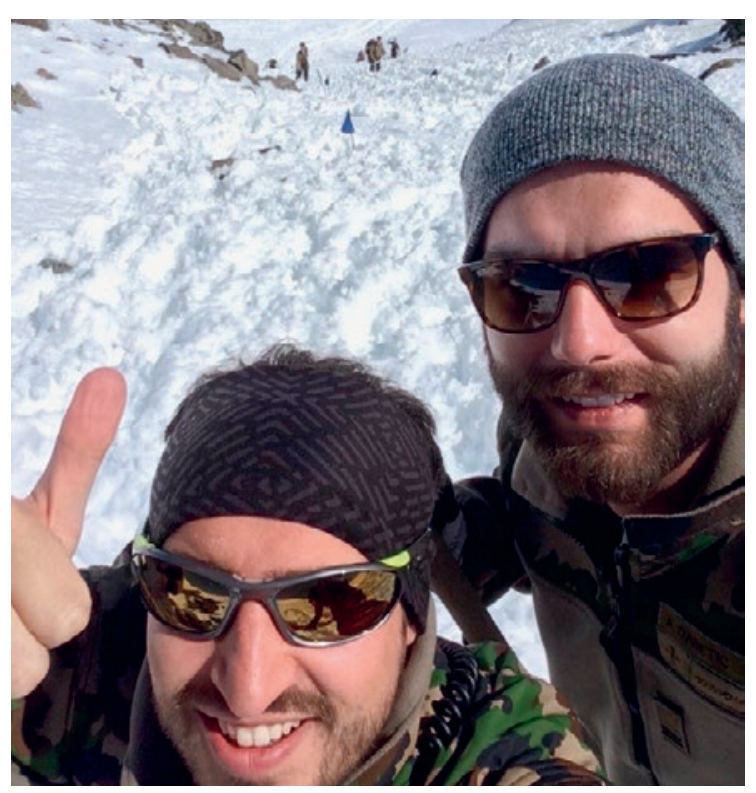

Oberleutnant Christophe Bianchi (links), Arzt am Patientensammelpunkt Rosablanche, PdG 2016, Facharzt für Innere Medizin und ISP für Notfallmedizin. 


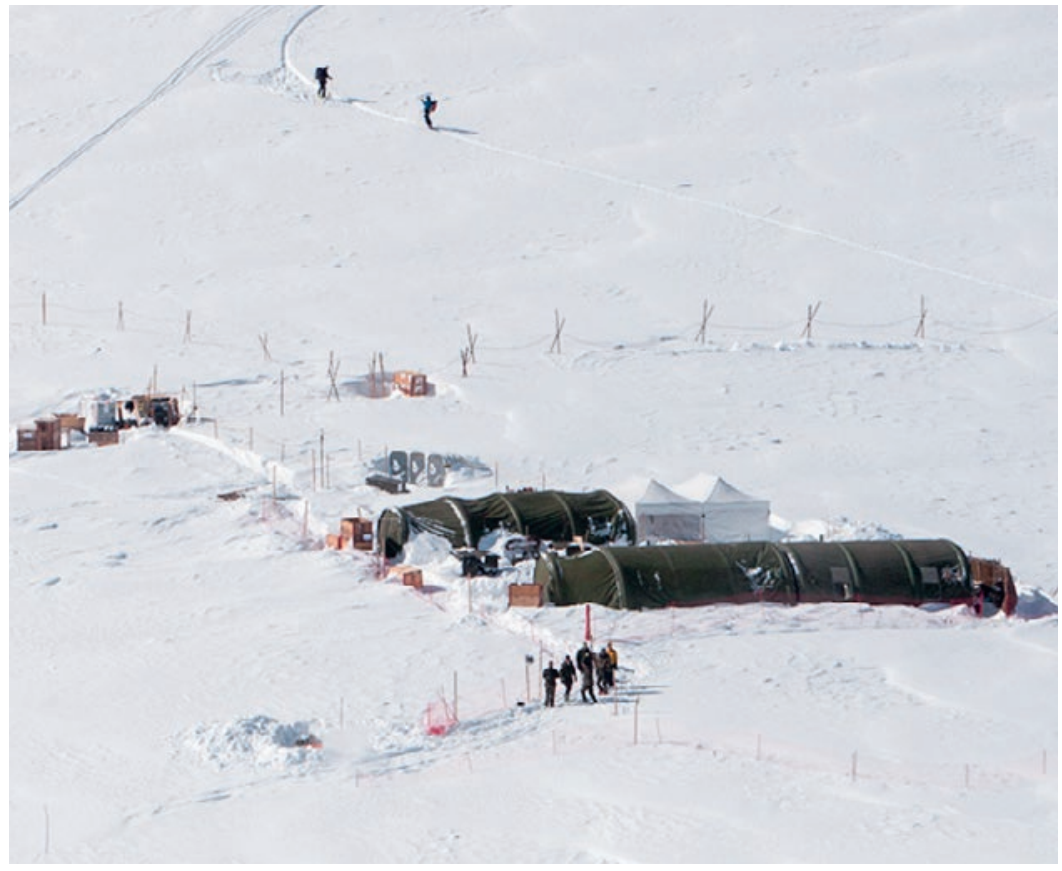

Abbildung 4: Vorbereitung der Strecke. Die ersten Paletten mit sanitärer Ausrüstung werden vier Wochen vor Beginn der PdG auf die Höhenposten geflogen. nische Fachkräfte, die motiviert sind, ab 2022 für mehrere Ausgaben der PdG mitzumachen. Eine Ausbildung in Anästhesie, Intensivmedizin, Notfallmedizin oder Traumatologie ist wünschenswert. Insbesondere Fachpersonal, das bereits Rettungsdienste geleistet hat und weiss, wie man sich im Hochgebirge verhält, wird eingeladen, sich zu bewerben. Es handelt sich um einen Echteinsatz unter reellen Bedingungen (Abb. 4). Die sanitätsdienstlichen Elemente müssen unter teilweise extremen Bedingungen jederzeit einsetzbar sein. Die Einberufung erfolgt durch freiwilligen Militärdienst oder durch einen zivilrechtlichen Vertrag für eine Gesamtdauer von acht Einsatztagen und einem Tag obligatorischer Weiterbildung.

\section{Bildnachweis}

Kommunikationsabteilung Stab Kdo PdG

\section{Literatur}

1 Weiss M, Bernoulli L, Zollinger A. The NACA scale. Construct and predictive validity of the NACA scale for prehospital severity rating in trauma patients. Anaesthesist. 2001 Mar;50(3):150-4.
Major Raphaël Kohlprath Stab Kommando Patrouille des Glaciers Rue du Catogne 7 CH-1890 Saint-Maurice pdg.tdiv1[at]vtg.admin.ch

\section{Das Wichtigste in Kürze}

- Seit mehr als einem halben Jahrhundert führt die Armee die Patrouille des Glaciers (PdG) durch, um ihre Truppen für die Bedingungen im Hochgebirge zu trainieren.

- Der Sanitätsdienst für diesen internationalen Wettbewerb ist beachtlich: 2018 waren 54 medizinische und 30 paramedizinische Fachkräfte im Einsatz.

- Bei jeder Ausgabe werden etwa 140 Verletzte und sechs Tonnen medizinisches Material mit dem Helikopter transportiert. Die häufigsten leichten Erkrankungen sind die akute Höhenkrankheit, leichte Atemwegserkrankungen, leichte Verdauungsstörungen, Erschöpfungszustände und leichte Knochen- und Gelenktraumata.

- Die PdG sucht motivierte Ärztinnen und Ärzte sowie paramedizinische Fachkräfte, die ab 2022 während mehrerer Ausgaben am Wettbewerb teilnehmen möchten. Eine Ausbildung in Anästhesie, Intensivmedizin, Notfallmedizin oderTraumatologie ist erwünscht. 